\title{
A Real Time Patient Monitoring System based on Artificial Neural Fuzzy Inference System (ANFIS)
}

\author{
Kajal Singh \\ Department of Mechanical and \\ Automation Engineering \\ Indira Gandhi Delhi Technical \\ University For women \\ Delhi, India
}

\author{
Divya Sharma \\ Department of Mechanical and \\ Automation Engineering \\ Indira Gandhi Delhi Technical \\ University For women \\ Delhi, India
}

\author{
Shipra Aggarwal \\ Department of Mechanical and \\ Automation Engineering \\ Indira Gandhi Delhi Technical \\ University For women \\ Delhi, India
}

\begin{abstract}
Over the last few years there has been tremendous growth in the field of healthcare monitoring systems in hospitals and outside of it. Developing wireless health care monitoring devices employing various technologies has become a keen area of interest in India and as well as in other Nations. This proposed work aims to integrate artificial neural intelligence in domain of healthcare monitoring. Wireless body sensor devices have the ability to reach an advance level of human body monitoring utilizing various transmission and data analytics techniques. Implementation of Artificial Neural Fuzzy Inference Systems (ANFIS) would enable the system to work as a smart healthcare system that decides the priority by itself based on the collected psychological parameters from the sensor nodes. Proposed model describes an e-healthcare monitoring system developed for realizing integration of ANFIS in healthcare monitoring systems. The model consists of sensors to collect vital data from patient's body which is then transmitted by Wi-Fi to a central HUB where fuzzy logic converts the raw data in linguistic variable which is trained in ANFIS to get the status of patient. The developed system provides the reliable, accurate and real-time accessible data of patients continuously and transmits the vital information using a dedicated communication module in case of emergency.
\end{abstract}

\section{Keywords}

Patient's vital signal monitoring; artificial neural fuzzy inference system (ANFIS); wireless transmission; GSM module.

\section{INTRODUCTION}

Healthcare monitoring always has the scope of improvement as it involves different machines and equipment to track a patient's health parameters continuously. Technology is improving, and so the healthcare applications. Healthcare is a critical science to deal with because it involves a human life which cannot be risked for lack of advanced healthcare devices. In this pursuit there are many e-healthcare devices developed employing various ideas and techniques. Healthcare ATMs and air ambulances are being launched in India and other Nations to compete with the fast growing demands of healthcare services during emergencies. A very challenging task in this research area, that has attached a lot of interest, is identifying the communication protocol and network architecture [1]. Continuous health care monitoring is necessary for the patients in hospitals or homes as well, so as to tack the patient's body parameters and provide a consistent and reliable data to the doctors or the medical staff for diagnosis. Such an approach is particularly beneficial to old, physically challenged and those people who live alone and not be able to seek help in case of medical emergencies. Ever increasing population and rise in the number of patients has led to shortage of staff resources in developing countries like India in the health care sector. Thus healthcare sector requires new models for information handling and communication in order to guarantee quality-oriented health care of the elderly [2]. The problem of elderly people is, they can't frequently visit the doctor or they can't move easily from place to place [3]. This can be resolved by integrating advanced communication technologies and data analytics in the healthcare monitoring such a model needs to be highly efficient, compact and low cost monitoring device.

\subsection{Wireless sensor network}

Wireless sensor network (WSN) is an emerging technology and has great potential to be employed in critical situations [4]. For this purpose various technologies is being adopted to generate a secure and reliable real-time patient monitoring. Wireless body area network is a special purpose wireless sensor network that incorporates different networks and wireless devices to enable remote monitoring for various environments $[5,6]$. The present system is based on several sensors connected together and shares a single wireless network. To increase the ongoing efforts in healthcare domain it is essential to develop, a low cost efficient monitoring device utilizing different types of sensors and latest technologies.

\subsection{Artificial Neural fuzzy inference system (ANFIS):}

For generating an intelligent system that can count on the patient's conditions regularly a system should be developed, so that human negligence can reduce upto a level. Artificial Neural Fuzzy Inference Systems is the combination of fuzzy logic and artificial neural network (ANN) that can logically generates fuzzy rules to make a system robust. A healthcare device has been developed using ANFIS technique and presented in this paper that tells about the patient's condition. The neural networks have the ability to adapt itself to the changes in input until the output matches the desired value increasing the reliability of the system. Hence a combination of fuzzy logic and adaptive nature of neural network is made use for detecting the health status of an individual [2]. The basic block diagram of fuzzy inference system is shown in figure1. 


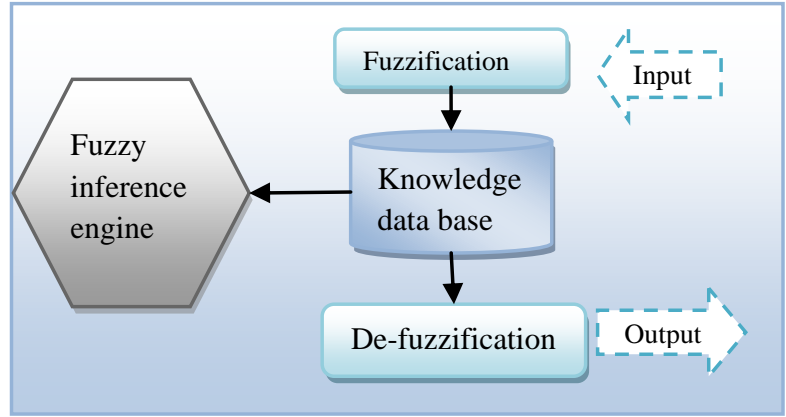

Fig 1: Units of fuzzy inference system

The figure1 consist of component of fuzzy inference system. It comprises various steps to train the inputs collected from the sensors. First step is fuzzification, in which raw data is collected and using fuzzy inference engine several rules are made artificially. These rules are then trained using sugeno method with the help of ANN. After completion of training the last step is de-fuzzification in which fuzzized data converted back into raw variables. This whole process minimizes human decision making process and also overcome human errors.

There is always a need to prioritize the condition of the patients in the hospitals as there are several patients to be diagnosed, and then it becomes difficult to decide which patient needs an intensive care first. To resolve this problem an intelligent system should be developed that can deal with the patient monitoring in real-time, so that a suitable and quick action can be taken to save a human life. For this Sugeno based artificial neural fuzzy inference system is used which is deciding the priority of patient according to fuzzy rules according to vital parameters collected from the device sensor.

The designed system is real time system, it collects real time data from the patient's body like pulse rate, body temperature and horizontal \& vertical position of patients and provides the updated status to the medical server, hospital staff and to the patient relatives using GSM module. This autonomous advance system fathomed the traditional methods to collects the patient vitals regularly by the hospital staff and nurse. It also resolves the human error in collecting the physiological parameters manually. This is fully automated advanced system in order to reduce fatalities occurring in the hospitals due to some delay or negligence. This paper describes two symmetrical portable sensor nodes employing Wi-Fi module to check the vital signs of two different patients at the same time and transmit the real time values to a central hub so that a comparison can be made to analyze which patient need an intensive care more than other patients.

The rest of the paper is structured as follows. Section II describes the literature review of existing technologies. Section III depicts the overview of the system architecture and discusses the adopted method to develop the system. In section IV analysis of results is discussed. The last section, section $\mathrm{V}$ concludes the paper and state the future scope of the developed healthcare device.

\section{LITERATURE REVIEW}

The quality of the health care is improving due to the advancement in biomedical science and technology [7]. Wireless healthcare devices have recently received more attention and interest in developing variety of application related to its various protocols for developing these devices. Some great research has been reviewed are as follows:

Authors in [8] developed an advanced health monitoring system for home based on adaptive neuro fuzzy inference system and also simulation results indicate that the performance of the ANFIS approach is suitable to develop the system and at the same time it is easy to implement. The developed healthcare system can be useful for the elderly and helpful to the pregnant women for their regular checkups without personally visiting to the clinic. In [1] researchers designed a network reliability event detection scheme for wireless sensor and actors network that is based on fuzzy logic system. Authors in [3] proposed a ubiquitous architecture of healthcare system. The system designed by [7] a fuzzy rules based fuzzy inference system for health monitoring using various sensors like pulse oximetry, temperature sensor, ECG sensor, nasal airflow sensor and heart beat sensor depending on their characteristics that defines the condition of patient according to the rule base. Authors in [9] developed a system which can target both monitoring of old people and for monitoring rehabilitation after hospitalization period.

A ZigBee based wearable physiological parameters monitoring system developed by [10] using impact sensor heart rate sensor and temperature sensor the prototype was successfully developed and algorithms were tested and found to be accurate and reliable. There are also many system has been developed to control the congestion control in communication of these health care devices like Sara Ghanavati et al., [11] developed a congestion control schemes based on fuzzy logic in wireless body area network. Researchers in [12] developed a cross-layer fuzzy logic scheduling algorithm to provide a highly reliable energy saving body sensor network for healthcare. Although various system employing several technologies providing great applications has been developed already, but still there is always a need to provide a more efficient and an intelligent system because of the limitations of the above developed system. Hence in order to deliver reliable and accurate results the proposed system has been designed that can also sets the priorities of the patient to save a person's life.

\section{OVERVIEW OF HEALTHCARE MONITORING UNIT}

The overview of the developed system is shown in figure 2 . The hardware and software together create an integrated automated environment to detect the priority of the patient's body condition. The system is having two sensor nodes to collect the physiological parameters of a patient's body like heart rate, body temperature and fall condition using accelerometer which is working as an impact sensor. Each sensor nodes collects and transmits the vital signal to a central hub using Wi-Fi as a dedicated communication device. At the central hub a Wi-Fi receiver accepts the sensor data in real time and transmits the data to the graphical user interface (GUI) in MATLAB. After gathering data, fuzzy logic converts the raw data in linguistic variable which is trained in ANFIS to get the status of patient. On the basis of patient condition the neural inference system sets the priority of patient's health status to get which patient needs intensive care more than others. The message is then sent through GSM regarding patient real time health condition to the hospital staff and medical servers. 


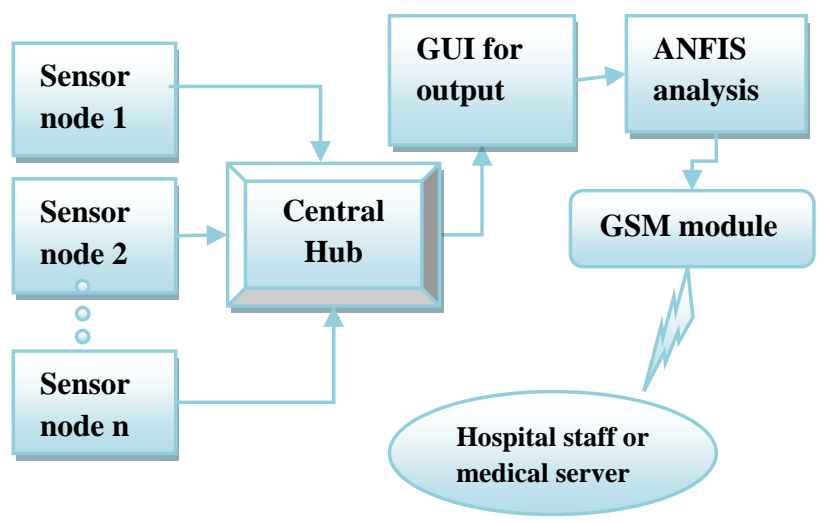

Fig 2: Overview of the system architecture

The overall system has been developed in two phase. The first phase is designing and development of hardware sensor nodes and second phase is MATLAB simulation phase, both are described below:

\subsection{Hardware development of sensor nodes}

In hardware development we have designed sensor node for each patient employing three sensors temperature sensor, accelerometer and pulse meter as shown in figure 3. ATmega 16 development board is used to embed the sensors and Wi-Fi module as a transmitter.

For body temperature measurement, LM35 temperature sensor is used in the designing of patient sensor node that gives an analog output, this voltage is measured by microcontroller using analog to digital converter (ADC). The LM35 gives its output in degree Celsius.

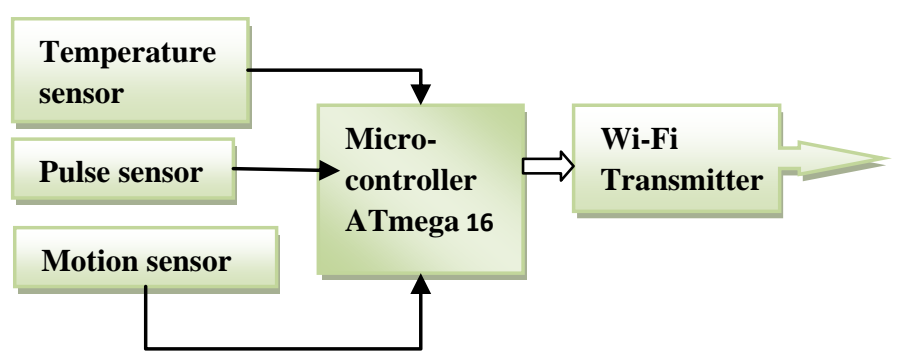

Fig 3: Block diagram of Patient sensor node

A pulse sensor is used to measure the rate of flow of blood in patient's body per minute which is also called as heart beat. Pulse sensor works on the principle of near-infrared spectroscopy (NIR). NIR involves using light in wavelength of $700-900 \mathrm{~nm}$ to measure blood volume [10]. At these wavelengths most of tissues do not absorb light other than hemoglobin which is actually required to monitor the pulse rate. It measures the volume change in the flow of blood using infra red LED. An Accelerometer (ADXL 213) was used to detect the body position. Its gives output in two-axis response horizontal vales and vertical values. It provides a digital voltage; the amplitude of voltage is directly proportional to the acceleration. MATLAB algorithm is used to detect sharp impacts, while ignoring slower moments like walking, sitting, sleeping, standing and writing etc.

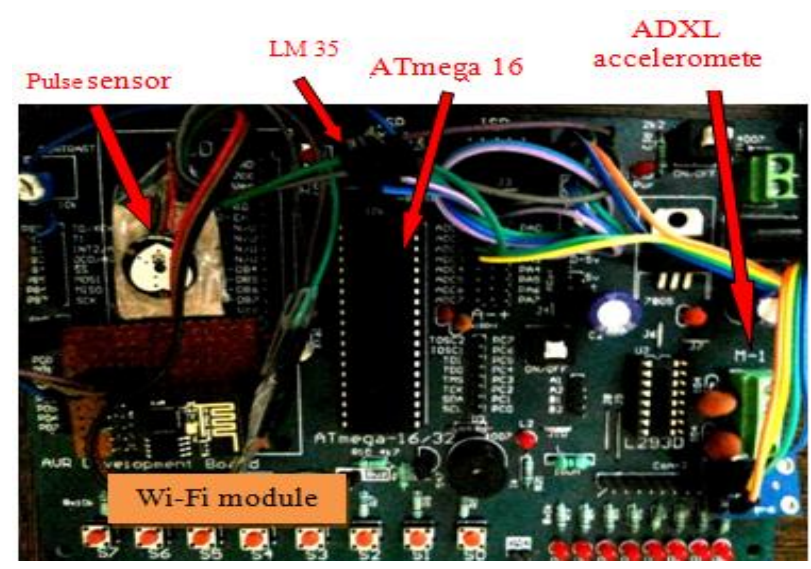

Fig 4: Prototype of system hardware unit

The prototype of the system hardware unit is shown in the figure 4. The two symmetrical prototype units (sensor node) are developed as shown in figure 4. Each one is comprised of one pulse sensor, one temperature sensor, motion sensor and one Wi-Fi module. The system is developed on ATmega16/32(microcontroller) development board. At portA all three sensors are interfaced and at portD Wi-Fi module is attached using USART, it connects the node to the server or central hub. At central hub a USB to TTL converter is connected that connects the Wi-Fi module to the system. After initializing all three Wi-Fi modules, server-client model is created where central hub is working as server and both sensor nodes worked as clients.

\subsection{MATLAB Simulation}

The simulation for the system has been done in MATLAB tool. First of all a graphical user interface (GUI) has been created to depicts the various parameters of the patient body and their graphs with reference to time as shown in figure5. Using Wi-Fi module a server has established between the terminal and sensor nodes where the terminal is working as a central hub and sensor nodes as a client. Then the central hub receives input from the sensor nodes and continuously displays the parameters of patient physiological parameters in real time using GUI. The graph of each parameter has plotted in GUI with respect to time. The graph shows the variation of different inputs in real time separately. Now this data is stored in FUZZY file and used for training in ANFIS. 

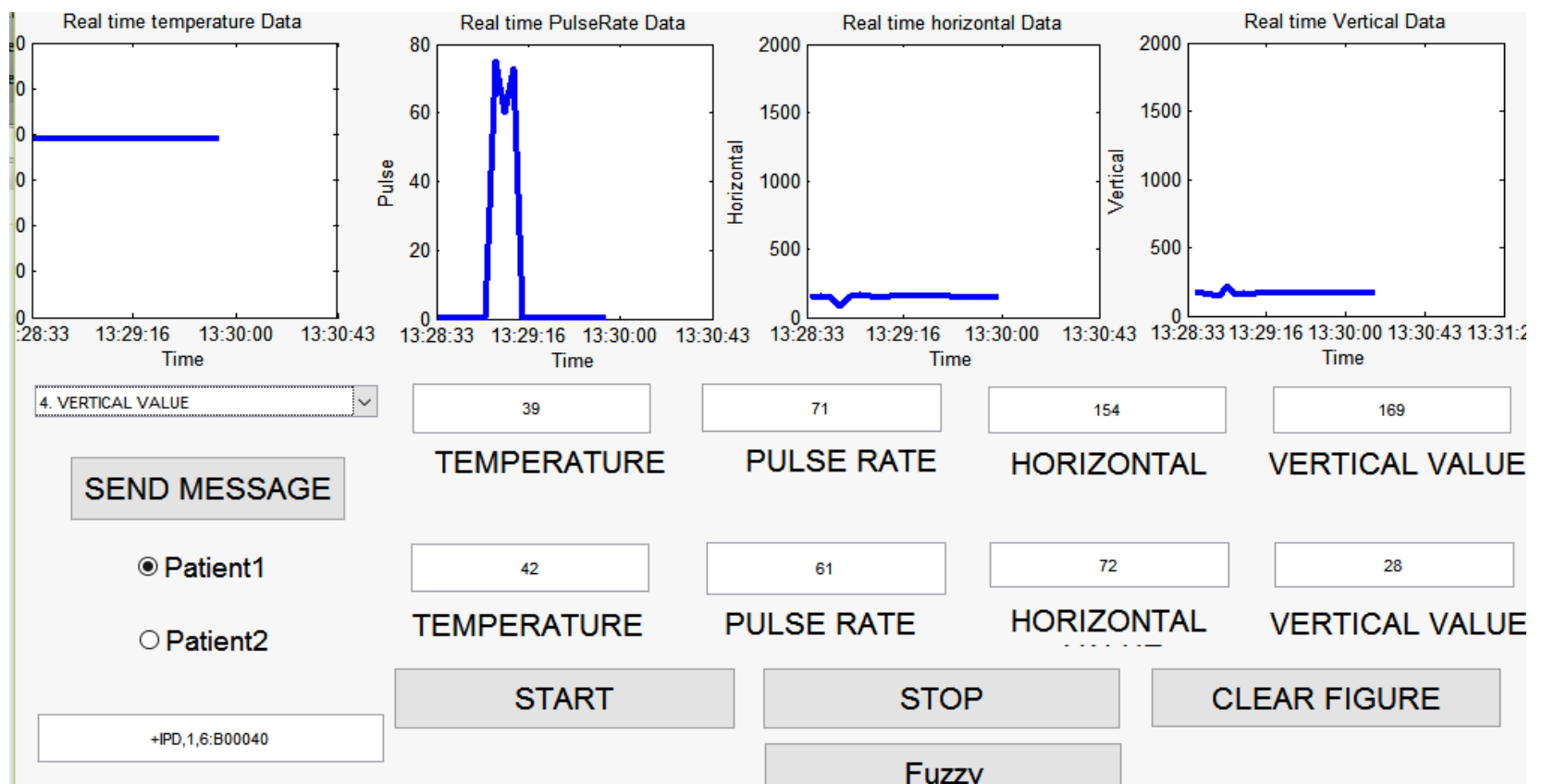

42

TEMPERATURE

PULSE RATE
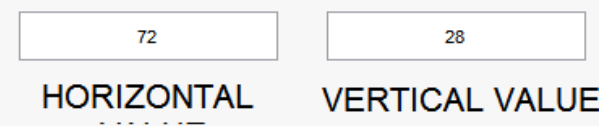

START

\begin{tabular}{|l|}
\hline STOP \\
\hline Fuzzy \\
\hline
\end{tabular}

\section{CLEAR FIGURE}

Fig 5: Real time GUI for patient monitoring

\section{IMPLEMENTATION OF ANFIS FOR HEALTH MONITORING}

ANFIS is a simple data learning technique that uses Fuzzy Logic to transform given inputs into a desired output through highly interconnected Neural Network processing elements and information connections, which are weighted to map the numerical inputs into an output [8]. So for this real time monitoring system fuzzy inference system in combination with highly connected neural network is used to obtained desired results. The system is checked for three vital input data obtained from GUI are collected and testing of our system for these four inputs is done using Gaussian form membership function. The fuzzy inference model, shown in figure 6 is developed using four membership functions.

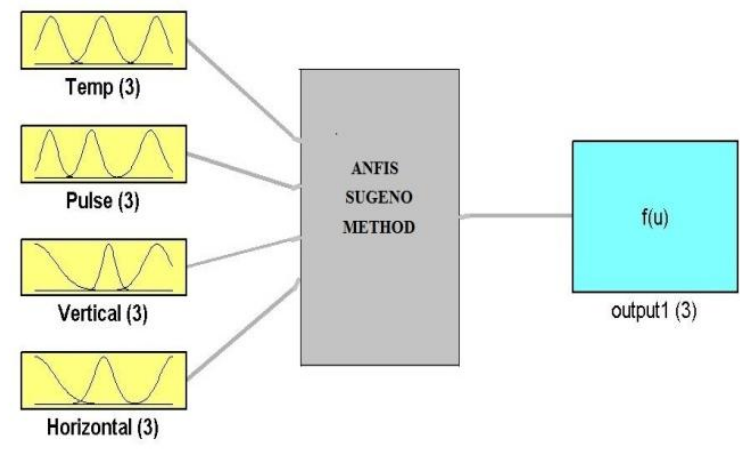

Fig 6: Fuzzy inference model using sugeno method

The each membership function is shown below in the figure7 for temperature variation, pulse rate, horizontal and vertical positions with reference to movement of patient.
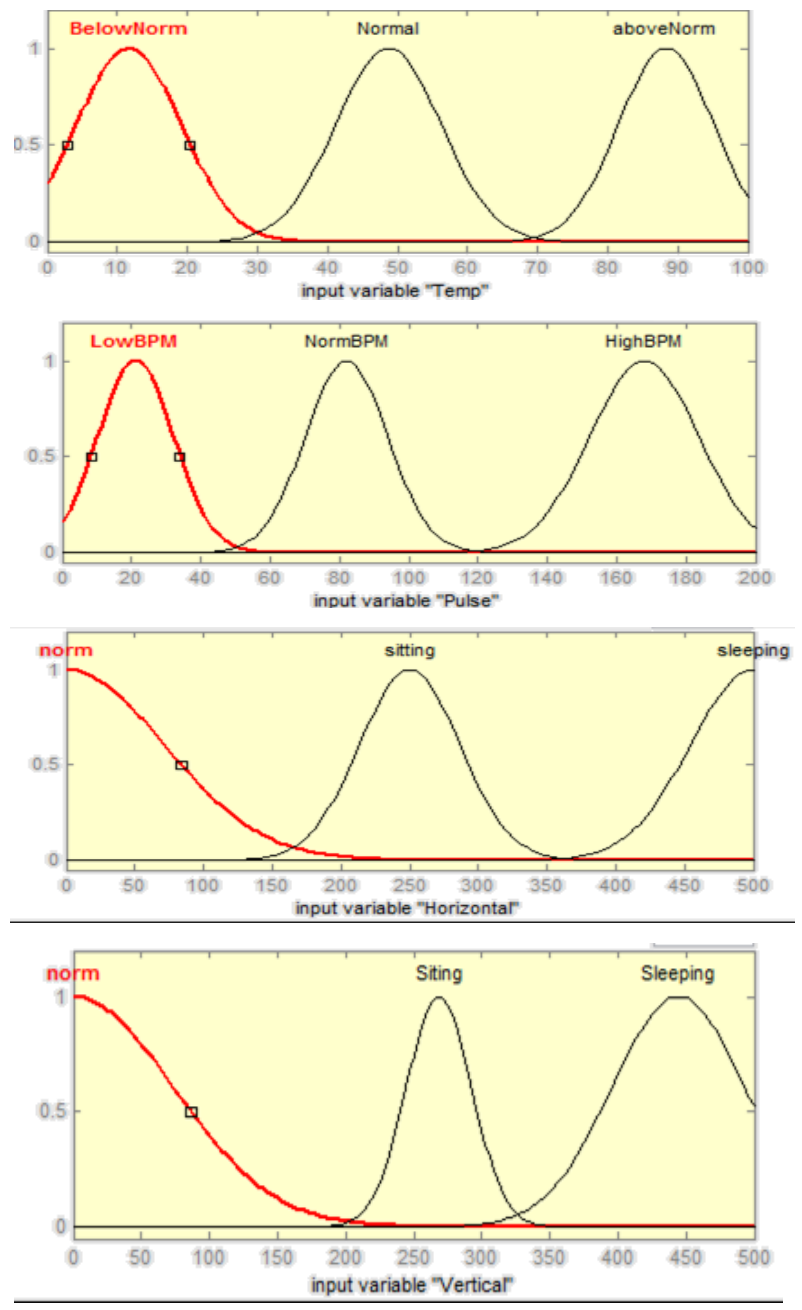

Fig 7: Input Membership functions (Temperature, pulse rate, horizontal position and vertical position) 
After defining the input membership function and target output the ANFIS system automatically generates the decision rules according to linguistic values of fuzzy system which is obtained after fuzzification process.

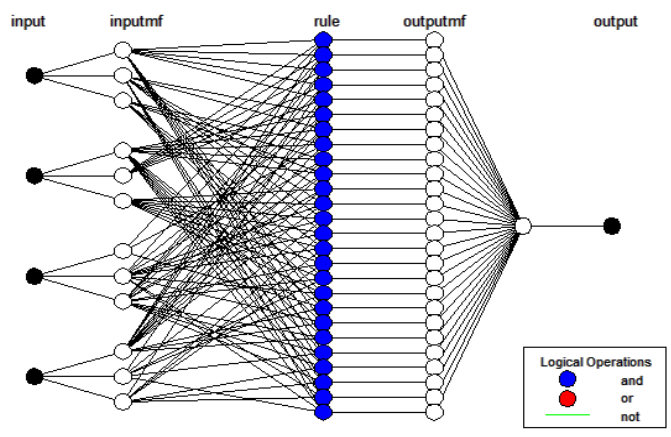

Fig 8: ANFIS training model

For implementation Sugeno method is used for training of fuzzy linguistic variables. Then training process starts and around 26 rules are generated for the mentioned Sugeno system which is shown in the ANFIS structure model (figure8). The generated ANFIS training model showing different condition of patients based on IF-THEN rules and AND logic which is shown in below figure using blue dots. The Sugeno system provides a single output stating the health status and then central hub transmits the status to the doctors and hospital staff. The advantage of ANFIS is that, different input cannot share the same output membership function.

\section{RESULTS AND DISCUSSION}

\subsection{Proteus simulation}

The simulation of system hardware is done in Proteus 8.1 tool using LM35 as a temperature sensor and for pulse sensor and accelerometer two potentiometer is used as shown in the figure 9. A schematic diagram is drawn on Proteus and then it was simulated for real time values to analyze the performance of hardware module.

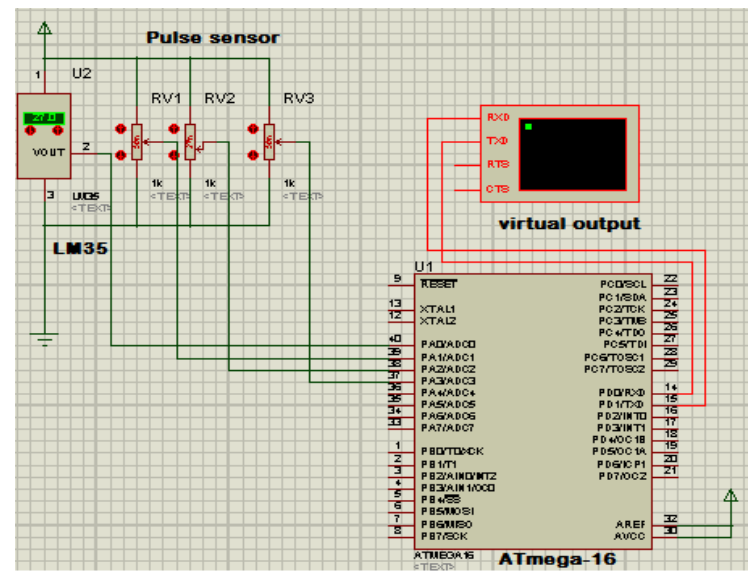

Fig 9: Proteus simulation of hardware component

\subsection{MATLAB ANFIS simulation}

With the help of ANFIS sugeno simulation method the system become intelligent and robust to state the health condition of a human body and also sets the priority. The fuzzy data set is divided in two sub set: the training data set and the testing data set. Training data set is used to train the fuzzy network of the monitoring system and to evaluate the response testing data set is used. The evaluation of all fuzzy rules is done by using AND or OR operators on all fuzzy sets. Now, the results from all fuzzy rules are evaluated and after evaluation these results are combined to obtain a final result which is called fuzzy output for the system. This process is termed as Inference of fuzzy. This fuzzified value is now converted to defuzzified value in order to obtain final output. Various experiments are conducted using training data set and to evaluate the accuracy of results, testing data set is used after completion of training process. The ANFIS rules are generated as shown in figure 10. This paper considers the following values to conduct the various experiments and to ensure the proper working of developed system.

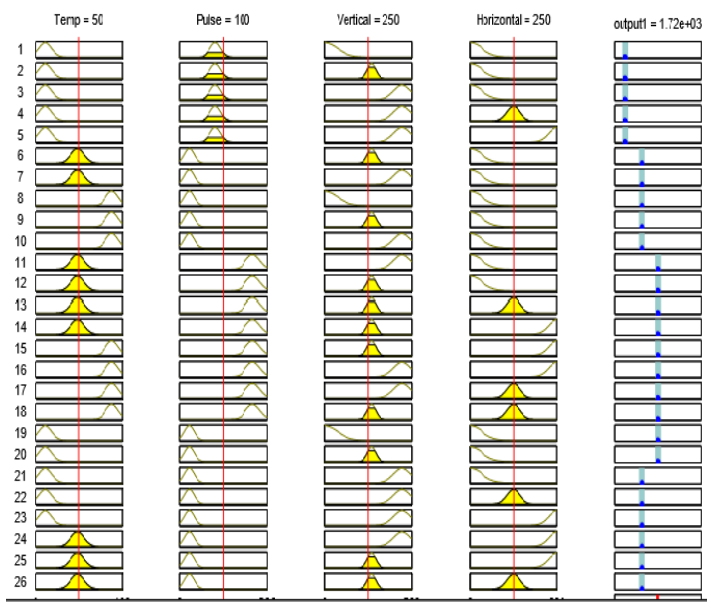

Fig 10: Fuzzy output with reference to input variations

The above parameters shows that the patient 2 is severely critical as fuzzy output is 1720 (see figure 10). In this situation a message is sent via GSM which is shown below:

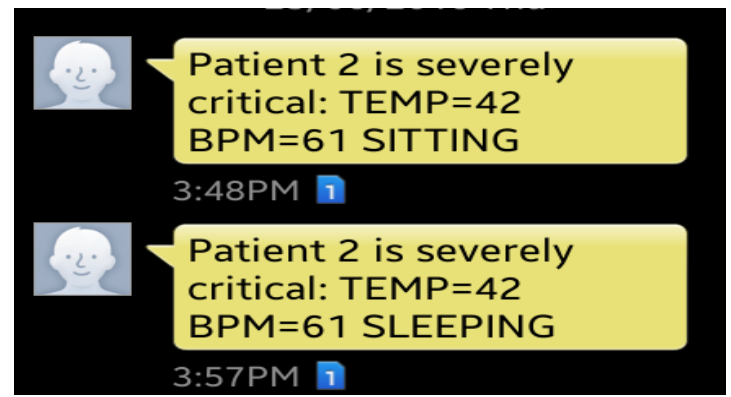

Fig.11. Mobile alerts sent to the doctors

After analysis of the input-output fuzzy membership function several surface 3D graphs has generated that shows the variation of the health status with reference to the changes in parameters at input sides.

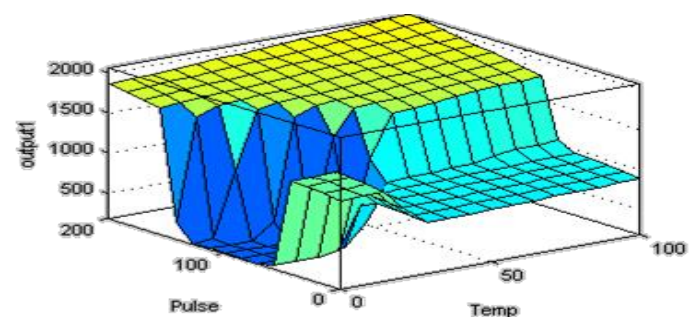

Fig 12: Surface plot of pulse rate v/s body temperature 


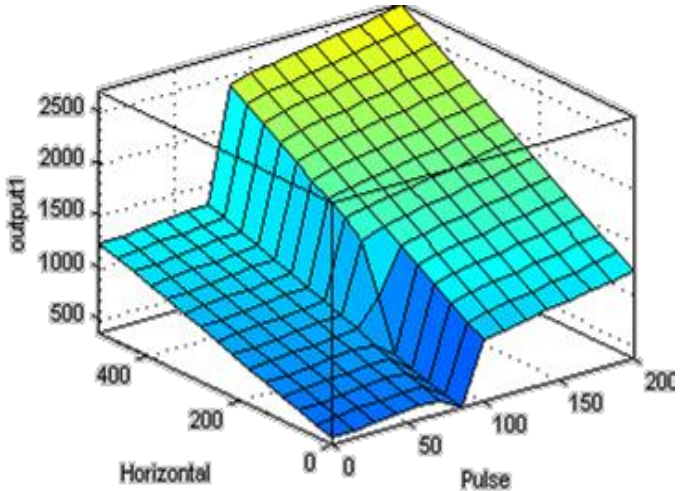

Fig 13: Surface plot of pulse rate v/s horizontal value

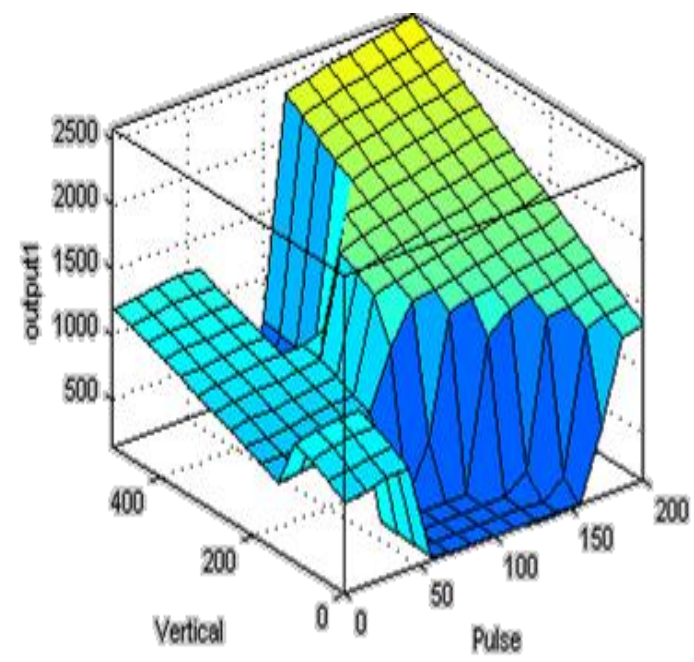

Fig 14: surface 3D plot of pulse rate v/s vertical value

Based on these surface graphs, the results are analyzed in tabular form which is given below:

Table.1.Experimental analysis of different parameters

\begin{tabular}{|l|l|l|l|}
\hline S.No. & Pulse sensor & Furzy output & Condition \\
\hline 1. & $0-55$ & $>1500$ & Critical \\
\hline 2. & $56-100$ & $<500$ & Normal \\
\hline 3. & $101-120$ & $<500$ & Normal \\
\hline 4. & 120 and above & $>1500$ & severely Critical \\
\hline S.No. & Temperature range & Furzy output & Condition \\
\hline 1. & $22-30$ & $1000-1500$ & Normal \\
\hline 2. & $31-38$ & $<500$ & critical \\
\hline 3. & Above 38 & $>1500$ & Severelycritical \\
\hline S.No. & Horizontal position & Vertical position & Person(posture \\
\hline 1. & & & \\
\hline 2. & $<200$ & $80-100$ & Sitting \\
\hline
\end{tabular}

\section{CONCLUSION AND FUTURE SCOPE}

This paper proposes an intelligent real-time patient monitoring system for hospitals and outside of it. The system generates an alert message after analysis of patient's vital body parameters in case of critical condition and emergency. By utilizing ANFIS architecture model the system becomes more reliable, efficient and robust. The proposed model can be enhanced researching more in mobile and lightweight body sensors to provide more accurate readings to fuzzy so that it provides a more accurate and reliable data for analysis. Further advancement in such a model could be achieved with implementation of another growing technology, Internet of Things (IOT). It would enable the system to talk to various other devices and interfaces ensuring better communication of analysis. The vision should be to improve the proposed model to possess Artificial Intelligence so that real time prescription could be given to the patient's using such a monitoring device.

\section{REFERENCES}

[1] Dima S.M., 2012 Antonopoulos C, Gialelis J, Koubias S, "A network reliability oriented event detection scheme for wireless sensor and actors networks", IEEE conference fuzzy system

[2] Mehmet R.Yuce, 2010 "Implementation of wireless body area networks for healthcare systems", Elsevier

[3] Kalyani K.L., Rajeshwari N, Yasaswani K, 2014 "WBAN: A Persuasive area in ubiquitous health care", vignana university Andhra Pradesh, India IJCSIT internation journal of computer science and information technologies, vol.5(1),60-63

[4] Padmavathi G, Shanmugapriya D and Kalaivani M, 2010 "A study on vehicle detection and tracking using wireless sensor networks", Scientific Research, Wireless Sensor Network, Vol.1, pp. 173-185

[5] WBAN standard group http://www.ieee802.org/15/pub/TG6.html,

[6] E. Jovanov, A. Milenkovic, C. Otto, P. Groen, 2005 “A wireless body area network of intelligent motion sensors for computer assisted physical rehabilitation" ,Journal of Neuro Engineering and Rehabilitation 2 (6)

[7] C.Billions R. K., Marck P. V., Dadious P, 2015 "Fuzzy inference system wireless body area network architechture simulation for health monitoring", IEEE International conference December 9-12

[8] Agrawal A.T. and Ashtankar P.S., 2013 “ Adaptive neuro-fuzzy inference system for health monitoring at home", International Journal of Advanced Science and Technology Vol. 55

[9] Zakrzewski M, Junnilal S, Vehkaojal A, Kailanto H, Vainio A.M., Defee I, Lekkalal J, Vanhala J and Hyttinen J, 2009 "Utilization of wireless sensor network for health monitoring in home environment", IEEE

[10] Malhi K, Mukhopadhyay S.C.,2015 “ A ZigBee based wearable physiological parameters monitoring system", IEEE sensor journal, vol 12

[11] Ghanavati S, Abawajy A and Izadi D, 2015 "A congestion control scheme based on fuzzy logic in wireless body area networks", $14^{\text {th }}$ IEEE conference on network computng and application

[12] Jamthe A,, Mishra A, Agrawal D.P., 2014 "Scheduling schemes for Interference Suppression in Healthcare Sensor Networks",IEEE ICC 
[13] Hamza N, Touati F, Khirji L, 2009 "wireless biomedical system design base on ZigBee technology for autonomous healthcare" ICCCP conference muscat

[14] Chen Y. M., Peng Y.J.,2013 "Energy Efficient Fuzzy Routing Protocol In Wireless Body Area Networks", vol. 4, ISSN2305-8269

[15] Bhunia S.S., Dhar S.K., Mukherjee N, 2014 "iHealth: A fuzzy approach for provisioning intelligent healthcare system in smart city", IEEE
[16] Ortiz A.M., Ababnch N, Timmon N, Morrison J, "Adptive Routing For Multihop IEEE 802.15.6 Wireless Body Area Network", IEEE conferenc

[17] Sudharakumar K B, Dhivya S, Mohanavalli S, Chnder R, 2015 "Cloud Based Fuzzy Healthcare System”, Procedia computer science

[18] Trucu C.E., Trucu C.O., 2013"Internet Of Things As Key Enabler For Sustainable Healthcare Delivery", Procedia computer science 